\title{
Evaluation of vancomycin therapy in the adult ICUs of a teaching hospital in southern Iran
}

This article was published in the following Dove Press journal: Drug, Healthcare and Patient Safety

\author{
Afsaneh Vazin \\ Motahare Mahi Birjand \\ Masoud Darake \\ Department of Clinical Pharmacy, \\ Faculty of Pharmacy, Shiraz University \\ of Medical Sciences, Shiraz, Iran
}

Background: Vancomycin resistance in intensive care units (ICUs) accounts for significant morbidity and excess costs. The objective of the present study was to determine the appropriateness of vancomycin use in the various ICUs of Nemazee Hospital, Shiraz, Iran.

Methods: This prospective study was performed on 95 critically ill patients (48 males and 47 females) who were treated with vancomycin for at least 3 subsequent doses in 6 ICUs during 12 months. Required demographic, clinical, and paraclinical data were collected by a pharmacist. Fifteen indexes were considered for evaluation of vancomycin use.

Results: Ventilator-associated hospital-acquired pneumonia (22.6\%), sepsis (22.1\%) and CNS infection $(12.6 \%)$ were found to be the most important indications for vancomycin prescription. Vancomycin was prescribed empirically in $81 \%$ of patients. None of the patients received loading dose, and most of the patients received fixed dose. The rate of prolonged empiric antibiotic therapy was $68.5 \%$ in patients who received vancomycin. The mean score of vancomycin use in the ICUs of Nemazee Hospital was $7.1 \pm 0.6$ out of 15 , implying that the rate of vancomycin use was in accordance with the guideline proposed by the Department of Clinical Pharmacy of Nemazee Hospital based on Infectious Diseases Society of America by $47.3 \%$.

Conclusion: Based on our results, the weakness in using vancomycin was related to not administering loading dose, the practice of prescribing fixed-dose vancomycin and prolonged duration of empiric therapy. Efforts to improve the pattern of vancomycin prescription and utilization in these ICUs should be undertaken.

Keywords: vancomycin, drug utilization, intensive care units

\section{Introduction}

Hospital-acquired infections (HAIs) are common in intensive care unit (ICU) patients and are correlated with increased morbidity and mortality. ${ }^{1}$ Currently, HAIs are a leading cause of death accounting for $78 \%$ deaths over the past two decades. Recent studies have revealed that nosocomial bloodstream infections are correlated with $35 \%$ mortality in ICU patients. ${ }^{1,2}$

Staphylococcus aureus and Staphylococcus epidermidis are the most common infectious causes in the ICUs. ${ }^{3}$ Staphylococcus infections are difficult to treat due to the rapid appearance of methicillin-resistant Staphylococcus aureus (MRSA) and methicillinresistant Staphylococcus epidermidis isolates (MRSE). ${ }^{4}$ MRSA and MRSE strains were first described in the middle of the 20th century and have since become endemic in many hospitals..$^{5}$ Approximately $5 \%$ of the patients in some health care institutions, especially in the US, were found to acquire MRSA colonization. Studies have also shown increased prevalence of methicillin resistance from $\sim 36 \%$ in 1992 to $\sim 64.5 \%$ in $2003 .{ }^{6}$ 
Vancomycin is the antibiotic of choice for most patients with suspected or confirmed MRSA or MRSE infections. Additionally, vancomycin is now recommended for treatment of patients with pneumococcal infections pending sensitivity results. ${ }^{3}$ An increasing number of high-risk patients, combined with increased bacterial resistance, have led to a remarkable increase in vancomycin use in some health care hospitals. ${ }^{7}$ Sadly, along with this increase in vancomycin use, resistance has increased among vancomycin-resistant Staphylococcus aureus, vancomycin-resistant Staphylococcus epidermidis and vancomycin-resistant enterococci (VRE), with the highest rates among isolates in ICU patients. ${ }^{8}$ Incidence of VRE in the ICUs showed a discrepancy among various regions and countries. For example, VRE was found to occur at a rate of 0.29 cases in 1000 patient ICU days in Germany, while a mean incidence of 27 cases in 1000 patient ICU days was reported in the US., ${ }^{9} 10$

Since the increased use of this antibiotic is considered one of the most important risk factors for infections in hospitals, research on vancomycin use can be an effective step towards the identification of problems related to prevention of vancomycin resistance. ${ }^{11}$ In this light, some studies have been conducted in this field in a variety of treatment centers. Nevertheless, more complete studies in this area may pave the way to reach the standard guidelines. Considering the lack of information about the use of vancomycin in ICUs from Nemazee Hospital, the present study aimed to evaluate the use of this antibiotic in the ICUs.

\section{Methods}

This prospective, cross-sectional study was conducted from May 2013 to May 2014 on patients admitted to ICUs of Nemazee Hospital, a general multispecialty, referral, tertiary health care setting, affiliated to Shiraz University of Medical Sciences, Shiraz, Iran. The study participants included all patients hospitalized in 6 ICUs of Nemazee Hospital (general ICU, central ICU, neurology ICUs 1 and 2, emergency ICU and surgical ICU) who received at least 3 consecutive fixed doses of vancomycin. Therefore, the patients receiving less than 3 consecutive fixed doses of vancomycin for any reason (including discharge, death, and treatment change and disruption of the antibiotic) were excluded from the study. Written informed consent was obtained from all participating patients or their proxies (if patient incapable of consent), and the study was approved by the Ethical Committee of Shiraz University of Medical Sciences. The data for this study were collected by a general pharmacist under the supervision of a clinical pharmacist.
Study data, including demographic characteristics (age, sex, height and weight), dates of admission and discharge, medical records, disease diagnosis, type of infection (HAIs or community-acquired infections), indication of vancomycin, laboratory parameters, creatinine clearance and method/ duration of vancomycin administration were collected using special forms. The data were analyzed to determine if the rate of vancomycin prescription was according to the guideline proposed by the Department of Clinical Pharmacy of Nemazee Hospital based on Infectious Diseases Society of America (IDSA) guideline. ${ }^{12}$ Prolonged vancomycin therapy was defined as empiric vancomycin therapy that was continued for at least 72 hours in patients who did not meet Centers for Control and Prevention guidelines (CDC) criteria for infection. ${ }^{13}$ It is noteworthy that the prevalence of infections caused by MRSA is reported to be $47.3 \%$ in our hospital.

Empiric antibiotics were used for suspected infections, eg, for ones not meeting CDC criteria or therapy had been continued for less than 72 hours.

To estimate the glomerular filtration rate, the most common methods utilized are measurement of the creatinine clearance and estimation equations based upon serum creatinine such as the Cockcroft-Gault equation and the Modification of Diet in Renal Disease (MDRD) study equation. ${ }^{14}$

Some indices were determined for assessment of vancomycin use and one score was assigned to each index. These indices included microbial culture before prescription, indication, prescription or non-prescription of the loading dose, dosing method, administration interval, preparation and dilution, duration of each injection (at least 1 hour), correct treatment period based on the type of infection, dose readjustment if necessary (including reduction of renal function), attention to serum creatinine and blood urea nitrogen levels before prescription of vancomycin, assessment of microbial cultures 48-72 hours after prescription of vancomycin, assessment of patients' serum creatinine levels every $2-3$ days, discontinuation or low doses of vancomycin in the patients whose serum creatinine level increased by more than $0.3 \mathrm{mg} / \mathrm{dL}$ in 48 hours, those whose serum creatinine level increased by 1.5 -fold compared with baseline in 7 days, and those whose urine output decreased to less than $0.5 \mathrm{~mL} / \mathrm{kg} / \mathrm{hr}$ for more than 6 hours, as well as high doses or changing the type of antibiotics in case of inappropriate responses to treatment. It is also worth mentioning that the patients' response to treatment was assessed based on fever, white blood cells (WBC) count, microbial cultures, consciousness levels, radiological images, and clinical features. A drug utilization evaluation (DUE) $\log$ sheet of vancomycin consisted of 15 variables. 
A score of 0 or 1 was given to each variable depending on whether the variable was evaluated as appropriate or inappropriate, respectively. This log sheet was completed for each patient. The total score was given to each patient by adding up the scores for each of the variables.

\section{Statistical analysis}

All statistical analyses were done using the SPSS statistical software (SPSS Inc., Chicago, IL, USA), version 15. Continuous and discrete variables were reported as mean \pm standard deviation and percentage, respectively.

\section{Results}

This study was conducted on 95 eligible critically ill patients during 12 months. Table 1 represents the patients' demographic

Table I Demographic and clinical information collected from the patients hospitalized in the ICUs of Nemazee Hospital, Shiraz $(\mathrm{n}=95)$

\begin{tabular}{|c|c|c|c|c|}
\hline \multicolumn{5}{|l|}{ Demographic and clinical data } \\
\hline Age, mean $\pm S D$ (range), year & \multicolumn{4}{|c|}{$19-88(15.6 \pm 48)$} \\
\hline Sex, male/female ratio & \multicolumn{4}{|c|}{$48 / 47$} \\
\hline Weight, mean \pm SD (range), kg & \multicolumn{4}{|c|}{$69 \pm 17.4(49-87)$} \\
\hline $\begin{array}{l}\text { Ideal body weight, mean } \pm \text { SD } \\
\text { (range), } \mathrm{kg}\end{array}$ & \multicolumn{4}{|c|}{$63.15 \pm 7.9(46-79.5)$} \\
\hline Height, mean \pm SD (range), cm & \multicolumn{4}{|c|}{$169.2 \pm 10.1(153-187)$} \\
\hline \multicolumn{5}{|l|}{ Infections, $\mathbf{N}(\%)$} \\
\hline Ventilator-associated pneumonia & \multicolumn{4}{|c|}{31 (22.63\%) } \\
\hline Sepsis & \multicolumn{4}{|c|}{$21(22.10 \%)$} \\
\hline CNS infection & \multicolumn{4}{|c|}{$12(12.63 \%)$} \\
\hline Meningitis & \multicolumn{4}{|c|}{$10(10.52 \%)$} \\
\hline Endocarditis & \multicolumn{4}{|c|}{$9(9.47 \%)$} \\
\hline Osteomyelitis & \multicolumn{4}{|c|}{$4(4.21 \%)$} \\
\hline Prosthetic joint infection & \multicolumn{4}{|c|}{$3(3.15 \%)$} \\
\hline Skin infection & \multicolumn{4}{|c|}{$3(3.15 \%)$} \\
\hline Catheter-associated infection & \multicolumn{4}{|c|}{$2(2.10 \%)$} \\
\hline \multicolumn{5}{|c|}{ Renal function before vancomycin initiation, $\mathbf{N}(\%)$} \\
\hline \multirow{2}{*}{$\begin{array}{l}\text { Clcr (Cockcroft and Gault), } \\
\mathrm{mL} / \mathrm{min} / 1.73 \mathrm{~m}^{2} \%\end{array}$} & $50(52$. & & & \\
\hline & $10-50$ & $0 \%)$ & & \\
\hline & $10(7.4 \%$ & & & \\
\hline Source of infection, $\mathbf{N}(\%)$ & & & & \\
\hline Community acquired & $44 \%$ & & & \\
\hline Nosocomial acquired & $56 \%$ & & & \\
\hline Treatment type, $\mathbf{N}(\%)$ & & & & \\
\hline Empirical & $81 \%$ & & & \\
\hline $\begin{array}{l}\text { Microbiologically documented, } \\
\text { (MRSA, MRSE)* }\end{array}$ & $19 \%$ & & & \\
\hline Vancomycin treatment perio & & & & \\
\hline Vancomycin treatment $\quad<5$ & $5-7$ & $8-10$ & $1 \mathrm{I}-20$ & $>20$ \\
\hline period & days & days & days & days \\
\hline Percent of patients & $11.5 \%$ & $24.3 \%$ & $38.9 \%$ & $21 \%$ \\
\hline
\end{tabular}

Notes: *MRSA and MRCoNS.

Abbreviations: ICUs, intensive care units; Clcr, creatinine clearance; MRSA, methicillin-resistant Staphylococcus aureus; MRSE, methicillin-resistant Staphylococcus epidermidis; MRCoNS, methicillin-resistant coagulase-negative staphylococci. parameters. Ventilator-associated hospital-acquired pneumonia (22.6\%), sepsis (22.1\%) and CNS infection (12.63\%) were detected to be the most important reasons for vancomycin prescription. As depicted in Table 1, it was found that $44 \%$ and $54 \%$ of the infections originated from community and hospital, respectively. Furthermore, vancomycin was prescribed empirically in $81 \%$ of the patients (Table 1). It is worth mentioning that none of the patients received loading dose and in most of the patients, vancomycin was prescribed as a fixed dose (ie, $1.0 \mathrm{~g}$ every 12 hours). Therapeutic drug monitoring was not used for any of the patients under investigation and dosage adjustments were determined traditionally. The rates of correctness of the prescribed dose of vancomycin using MDRD and Cockcroft-Gault equations are shown in Table 2. Results from our study also indicated that drug interval had to be adjusted for $43 \%$ of the patients under vancomycin treatment. However, this was performed only for $14 \%$ of the patients. In addition, approximately $24 \%$ of the study population experienced nephrotoxicity, the majority of whom received aminoglycosides and colistimethate sodium simultaneously (Table 3). In fact, no further treatment was carried out for $76 \%$ of the patients with increased serum creatinine levels. For the rest of the patients, vancomycin was discontinued or administered with longer intervals. Overall, the most common clinical and paraclinical issues noticed through vancomycin treatment periods included fever $(81 \%)$ and WBC count (64\%). WBC counts were found to be greater than $10,000 \mu \mathrm{L}$ in $60 \%$ of the study population at

Table 2 Percentage of correct and incorrect prescribed dose of vancomycin based on renal function estimates using MDRD and Cockcroft and Gault equations in the ICUs of Nemazee Hospital, Shiraz

\begin{tabular}{lllll}
\hline $\begin{array}{l}\text { Renal function } \\
\text { estimates }\end{array}$ & Clcr & $\begin{array}{l}\text { Under } \\
\text { dose (\%) }\end{array}$ & $\begin{array}{l}\text { Desirable } \\
\text { (\%) }\end{array}$ & $\begin{array}{l}\text { Overdose } \\
\text { (\%) }\end{array}$ \\
\hline Cockcroft and & $<10$ & 0 & 100 & 0 \\
Gault & $10-50$ & 23.81 & 76.19 & 0 \\
& $>50$ & 0 & 100 & 0 \\
MDRD & $<10$ & 0 & 100 & 0 \\
& $10-50$ & 42.11 & 57.89 & 0 \\
& $>50$ & 0 & 100 & 0 \\
\hline
\end{tabular}

Abbreviations: MDRD, Modification of Diet in Renal Disease; ICUs, intensive care units; Clcr, creatinine clearance.

Table 3 Drug-drug interaction with vancomycin that may lead to increased nephrotoxicity in the study population $(n=95)$

\begin{tabular}{ll}
\hline Drug-drug interaction & $\begin{array}{l}\text { Percentage of } \\
\text { total cases }\end{array}$ \\
\hline Vancomycin + amikacin & $12.63 \%$ \\
Vancomycin + colistin & $5.26 \%$ \\
Vancomycin + piperacillin tazobactam & $5.26 \%$ \\
Vancomycin + amphotericin B & $1.05 \%$ \\
\hline
\end{tabular}


the beginning of vancomycin treatment, followed by a $27.4 \%$ decrease at the end of the treatment. Additionally, culture results were noticed only in $26 \%$ of the patients. Moreover, $22 \%$ of the patients failed to show the appropriate response to the treatment, $56 \%$ of whom did not receive the necessary measurements. The treatment period lasted for 11-20 days in $38.9 \%$, less than 5 days in $4.3 \%$, and more than 20 days in $21 \%$ of the cases (Table 1). Overall the rate of prolonged empiric antibiotic therapy was $68.5 \%$ in patients receiving vancomycin.

In addition, the mean score of vancomycin use was $7.1 \pm 0.6$ out of 15 in the ICUs of Nemazee Hospital, indicating that vancomycin use was in accordance with the guideline proposed by the Department of Clinical Pharmacy of Nemazee Hospital based on Infectious Diseases Society of America by $47.3 \%$. Table 4 shows the accordance of vancomycin use in the ICUs of Nemazee Hospital.

\section{Discussion}

The evaluation programs of antibiotic use play an important role in the improvement of antibiotic therapy. This is because the understanding of the problems helps derive practice changes and improves the pattern of antimicrobial use. Based on findings from this study, $47.3 \%$ of the vancomycin use was consistent with the guideline.

The present study demonstrated that vancomycin was prescribed empirically in $81 \%$ of the cases, while prescribed in only $19 \%$ of the cases after microbial culture documentations. In a study in 2001 , You et al found that $46 \%$ of the empirical prescriptions were appropriate in a hospital in Hong Kong. ${ }^{15}$ Results from an investigation, performed in hematology- oncology ward of Nemazee Hospital in 2011, also indicated that vancomycin use was inconsistent with the guideline in $68.63 \%$ of the cases. ${ }^{16}$ Another study conducted in a teaching hospital in Tabriz in 2012 revealed that $69.3 \%$ of the patients received vancomycin inappropriately. ${ }^{17}$ In addition, some authors reported $65 \%$ of inappropriate empiric vancomycin prescription, according to the CDC. ${ }^{18,19}$ Our data have shown that the rate of empirical vancomycin prescription was higher in our study hospital, which might be attributed to different types of wards and prevalent infections. Ventilator-associated pneumonia and sepsis were the most prevalent indications for empirical vancomycin use in our ICUs. It should be noted that empiric antimicrobial therapy using one or more antimicrobials within 1 hour of presentation of sepsis is extremely important. Based on the 2016 IDSA/American Thoracic Society guidelines on hospital acquired pneumonia (HAP) and ventilator associated pneumonia (VAP) and the 2011 IDSA guidelines for the treatment of MRSA infections, either linezolid or vancomycin is recommended for infections suspected or proven due to MRSA. ${ }^{20,21}$ Therefore, sepsis and ventilator-associated pneumonia are reasons for high empirical vancomycin therapy in our study. The high rate of empiric vancomycin therapy in this study suggests that prolonged empiric antibiotic use is commonplace in our ICUs, which was confirmed by our results. It should be noted such a practice may be harmful in the absence of confirmed infections. ${ }^{22}$

We also showed that origin of infection was from the hospital (56\%) and the community (44\%). A study performed in Thailand in 2006 indicated that the prevalence of hospital infections was $4.9 \%{ }^{23}$ In addition, the prevalence of hospital

Table 4 Fifteen indices of vancomycin use in the study population $(n=95)$

\begin{tabular}{|c|c|c|c|}
\hline No & Index & $\begin{array}{l}\text { Appropriatel } \\
\text { performed, } \\
\text { percent }\end{array}$ & $\begin{array}{l}\text { Inappropriatel } \\
\text { not performed, } \\
\text { percent }\end{array}$ \\
\hline $\mathrm{I}$ & Indication & 74 & 26 \\
\hline 2 & Duration of injection & 100 & 0 \\
\hline 3 & Administration of a loading dose & 0 & 100 \\
\hline 4 & Maintenance daily dose & 76 & 24 \\
\hline 5 & Administration time interval & 13 & 87 \\
\hline 6 & Administration time interval after first dose & 23 & 77 \\
\hline 7 & Method of preparation and dilution & 0 & 100 \\
\hline 8 & Duration of treatment & 31.5 & 68.5 \\
\hline 9 & Evaluation of renal function estimates of the patients before vancomycin prescription & 89 & II \\
\hline 10 & Dose readjustment based on serum creatinine level & 14 & 86 \\
\hline II & Evaluation of microbial culture $48-72$ hours after vancomycin prescription & 42 & 58 \\
\hline 12 & Evaluation of serum creatinine levels periodically during treatment & 100 & 0 \\
\hline 13 & Take necessary measures (disruption or dose reduction) for patients who developed nephrotoxicity & 25 & 75 \\
\hline 14 & Take necessary measures for patients who had not responded to the treatment & 44 & 56 \\
\hline 15 & Administration route & 100 & 0 \\
\hline
\end{tabular}


infections was reported to be $48.7 \%$ and $11.9 \%$ in ICUs in Turkey and Kuwait, respectively. ${ }^{24,25}$ Thus, the incidence rate of infections was higher in Nemazee Hospital, which might be due to inattention to health protocols, particularly hand hygiene, in this hospital.

In serious gram-positive infections treated with vancomycin, especially in critically ill patients, administration of loading dose is necessary to improve early achievement of therapeutic vancomycin levels. ${ }^{26}$ In the present study, none of our patients was given the loading dose of vancomycin. This may be partially because of physician's unfamiliarity with loading dose administration.

In our study, the initial dose of vancomycin was prescribed in all patients as a fixed dose. Other studies showed that fixeddose vancomycin prescription, instead of weight-based dose, is a major reason for underdosing. ${ }^{27}$ Considering this fact, it is recommended to calculate the dose based on actual body weight and adjust subsequent doses based on therapeutic drug monitoring.

In ICUs of Nemazee Hospital, vancomycin intravenous infusion was prescribed for 60 minutes, which is consistent with vancomycin prescription guidelines. However, the injected solution's concentration was wrong in all the patients under the present investigation based on reference standards. According to the results, $10 \mathrm{mg} / \mathrm{mL}$ instead of $5 \mathrm{mg} / \mathrm{mL}$ concentration was injected to the patients.

Numerous studies suggested that more intensive vancomycin dosing schedules are associated with increased rates of nephrotoxicity. ${ }^{4,8}$ Some studies of contemporary vancomycin formulations have observed an acute decline in renal function associated with vancomycin monotherapy in 5-15\% of the patients. ${ }^{28-31}$ The variation between rates of vancomycin nephrotoxicity can be due to different criteria used for the definition of nephrotoxicity, different study populations and co-administration of nephrotoxic agents. In most guidelines, nephrotoxicity has been defined as a $0.5 \mathrm{mg} / \mathrm{dL}$ increase in serum creatinine if the baseline serum creatinine was $\leq 3 \mathrm{mg}$ / $\mathrm{dL}$, or a rise of $>1 \mathrm{mg} / \mathrm{dL}$ if the baseline serum creatinine was $>3 \mathrm{mg} / \mathrm{dL} .{ }^{8}$ Based on the abovementioned definition, in our study, $24 \%$ of the patients showed a significant increase in their serum creatinine levels. It should be mentioned that aminoglycosides and other nephrotoxic agents were simultaneously used in such patients.

In our study, $3.1 \%$ of the patients showed complications, such as red man syndrome together with itching, skin rashes, and back and chest pain, leading to disruption of vancomycin administration. Similarity, Geraci et al reported the preva- lence of rash and fever in 5\% of the patients. ${ }^{32}$ Furthermore, in the study carried out by Woodley and Hall, phlebitis was detected in nearly $30 \%$ of the patients. ${ }^{33}$

In this study, 15 indexes that influence appropriate vancomycin use were evaluated. Various indexes such as appropriate indication, selection of the correct dose, correct administration, interval between doses, obtaining culture before antibiotic and duration of therapy are important factors that should be considered. In our study, the sum of indexes of vancomycin use were relevant to standard guidelines in $43.7 \%$. However, this score is not the actual score of vancomycin in our population because scoring all the mentioned indexes for the whole population was not feasible.

One also should not overlook the fact that the most frequent weakness in using vancomycin was related to not administering the loading dose, the practice of prescribing fixed-dose vancomycin, prolonged courses of empiric therapy, incorrect method of reconstitution and not evaluating microbial culture 48-72 hours after vancomycin prescription. However, the treatment team's strong points in using vancomycin were accordance with indication, duration of each injection and daily measurement of the patients' serum creatinine levels.

It should be also noted that all the gathered data were not discussed due to the study's small sample size. Hence, larger sample sizes are required to obtain more reliable results. In addition, the patients had to receive at least 3 doses of vancomycin to be included in our study; hence, it may not reflect the real denominator and appropriateness of vancomycin use in our hospital.

In summary, the results of current study in the ICUs of Nemazee Hospital, Shiraz, Iran, indicated that administration of the loading dose is one of the issues that is apparently not taken into account. Measurement of serum vancomycin concentration was not performed in this hospital. In addition, treatment duration was another issue that has received little attention. It seems that consumption control and training programs are required to improve the process of using vancomycin in ICUs of this hospital.

\section{Acknowledgments}

This research was performed by Masoud Darake in partial fulfillment of the requirements for certification as a pharmacist. The present paper was adopted from the proposal number 95-01-36-12698 approved by vice-chancellor for research affairs of Shiraz University of Medical Sciences. 


\section{Disclosure}

The authors report no conflicts of interest in this work.

\section{References}

1. Haddadin AS, Fappiano SA, Lipsett PA. Methicillin resistant Staphylococcus aureus (MRSA) in the intensive care unit. Postgrad Med J. 2002;78(921):385-392.

2. Lucet JC, Chevret S, Durand-Zaleski I, Chastang C, Régnier B; Multicenter Study Group. Prevalence and risk factors for carriage of methicillin-resistant Staphylococcus aureus at admission to the intensive care unit: results of a multicenter study. Arch Intern Med. 2003;163(2):181-188.

3. Wright SW, Wrenn KD. Appropriateness of vancomycin use in the emergency department. Ann Emerg Med. 1998;32(5):531-536.

4. Hazlewood KA, Brouse SD, Pitcher WD, Hall RG. Vancomycinassociated nephrotoxicity: grave concern or death by character assassination? AM J Med. 2010;123(2):182.e1-e7.

5. Gill SR, Fouts DE, Archer GL, et al. Insights on evolution of virulence and resistance from the complete genome analysis of an early methicillin-resistant Staphylococcus aureus strain and a biofilm-producing methicillin-resistant Staphylococcus epidermidis strain. J Bacteriol. 2005;187(7):2426-2438.

6. Michel M, Gutmann L. Methicillin-resistant Staphylococcus aureus and vancomycin-resistant enterococci: therapeutic realities and possibilities. Lancet. 1997;349(9069):1901-1906.

7. Ena J, Dick RW, Jones RN, Wenzel RP. The epidemiology of intravenous vancomycin usage in a university hospital. A 10-year study. JAMA. 1993;269(5):598-602.

8. Van Hal SJ, Paterson DL, Lodise TP. Vancomycin-induced nephrotoxicity in troughs of " $15-20$ " $\mathrm{mg} / \mathrm{L}$ era: a systematic review and metaanalysis. Antimicrob Agents Chemother. 2012:AAC-01568.

9. Kohlenberg A, Schwab F, Meyer E, Behnke M, Geffers C, Gastmeier P. Regional trends in multidrug-resistant infections in German intensive care units: a real-time model for epidemiological monitoring and analysis. J Hosp Infect. 2009;73(3):239-245.

10. Warren DK, Kollef MH, Seiler SM, Fridkin SK, Fraser VJ. The epidemiology of vancomycin-resistant Enterococcus colonization in a medical intensive care unit. Infect Control Hosp Epidemiol. 2003;24(4): $257-263$.

11. Ingram PR, Lye DC, Tambyah PA, Goh WP, Tam VH, Fisher DA. Risk factors for nephrotoxicity associated with continuous vancomycin infusion in outpatient parenteral antibiotic therapy. JAntimicrob Chemother. 2008;62(1):168-171.

12. Rybak M, Lomaestro B, Rotschafer JC, et al. Vancomycin therapeutic monitoring: review and recommendations from the ASHP, IDSA and SIDP Task Force. Am J Health-Syst Pharm. 2009;66:82-98.

13. Recommendations for preventing the spread of vancomycin resistance. Recommendations of the Hospital Infection Control Practices Advisory Committee (HICPAC). MMWR Recomm Rep. 1995;44(RR-12):1-13.

14. Levey AS, Coresh J, Greene T, et al; Chronic Kidney Disease Epidemiology Collaboration. Using standardized serum creatinine values in the modification of diet in renal disease study equation for estimating glomerular filtration rate. Ann Intern Med. 2006;145(4): $247-254$.
15. You JH, Lyon DJ, Lee BS, Kwan SM, Tang HY. Vancomycin utilization at a teaching hospital in Hong Kong. Am J Health Syst Pharm. 2001;58(22):2167-2169.

16. Vazin A, Japoni A, Shahbazi S, Davarpanah MA. Vancomycin utilization evaluation at hematology-oncology ward of a teaching hospital in Iran. Iran J Pharm Res. 2012;11(1):163-170.

17. Hamishehkar H, Ebrahimi D, Mahmoodpoor A, Mashayekhi S, Asgharian P, Reazee H. Drug utilization evaluation of vancomycin in a teaching hospital in Tabriz-Iran. Pharm Sci. 2015;21(1):25.

18. Johnson SV, Hoey LL, Vance Bryan K. Inappropriate vancomycin prescribing based on criteria from the Centers for Disease Control and Prevention. Pharmacotherapy. 1995;15(5):579-585.

19. Evans ME, Kortas KJ. Vancomycin use in a university medical center: comparison with hospital infection control practices advisory committee guidelines. Infect Control Hosp Epidemiol. 1996;17(6):356-359.

20. Kalil AC, Metersky ML, Klompas M, et al. Management of adults with hospital-acquired and ventilator-associated pneumonia: 2016 clinical practice guidelines by the Infectious Diseases Society of America and the American Thoracic Society. Clin Infect Dis. 2016;63(5):e61-e111.

21. Liu C, Bayer A, Cosgrove SE, et al. Clinical practice guidelines by the Infectious Diseases Society of America for the treatment of methicillinresistant Staphylococcus aureus infections in adults and children. Clin Infect Dis. 2011;52(3):e18-e55.

22. Aarts MA, Brun-Buisson C, Cook DJ, et al. Antibiotic management of suspected nosocomial ICU-acquired infection: does prolonged empiric therapy improve outcome? Intensive Care Med. 2007;33(8):1369-1378.

23. Danchaivijitr S, Judaeng T, Sripalakij S, Naksawas K, Plipat T. Prevalence of nosocomial infection in Thailand 2006. J Med Assoc Thai. 2007;90(8):1524-1529.

24. Erdem H, Inan A, Altındis S, et al. Surveillance, control and management of infections in intensive care units in Southern Europe, Turkey and Iran-a prospective multicenter point prevalence study. $J$ Infect. 2014;68(2):131-140.

25. Salama MF, Jamal WY, Mousa HA, Al-Abdulghani KA, Rotimi VO. The effect of hand hygiene compliance on hospital-acquired infections in an ICU setting in a Kuwaiti teaching hospital. J Infect Public Health. 2013;6(1):27-34.

26. Truong J, Levkovich BJ, Padiglione AA. Simple approach to improving vancomycin dosing in intensive care: a standardised loading dose results in earlier therapeutic levels. Intern Med J. 2012;42(1):23-29

27. Hall RG 2nd, Payne KD, Bain AM, et al. Multicenter evaluation of vancomycin dosing: emphasis on obesity. Am J Med. 2008;121(6):515-518.

28. Appel GB, Given DB, Levine LR, Cooper GL. Vancomycin and the kidney. Am J Kidney Dis. 1986;8(2):75-80.

29. Bailie GR, Neal D. Vancomycin ototoxicity and nephrotoxicity. Med Toxicol Adverse Drug Exp. 1988;3(5):376-386.

30. Downs NJ, Neihart RE, Dolezal JM, Hodges GR. Mild nephrotoxicity associated with vancomycin use. Arch Intern Med. 1989;149(8):1777-1781.

31. Rybak MJ, Albrecht LM, Boike SC, Chandrasekar PH. Nephrotoxicity of vancomycin, alone and with an aminoglycoside. $J$ Antimicrob Chemother. 1990;25(4):679-687.

32. Geraci JE, Nichols DR, Wellman WE. Vancomycin in serious staphylococcal infections. Arch Intern Med. 1962;109(5):507-515.

33. Woodley DW, Hall WH. The treatment of severe staphylococcal infections with vancomycin. Ann Intern Med. 1961;55(2):235-249.
Drug, Healthcare and Patient Safety

\section{Publish your work in this journal}

Drug, Healthcare and Patient Safety is an international, peer-reviewed open access journal exploring patient safety issues in the healthcare continuum from diagnostic and screening interventions through to treatment, drug therapy and surgery. The journal is characterized by the rapid reporting of reviews, original research, clinical, epidemiological and

\section{Dovepress}

post-marketing surveillance studies, risk management, health literacy and educational programs across all areas of healthcare delivery. The manuscript management system is completely online and includes a very quick and fair peer-review system. Visit http://www.dovepress.com/ testimonials.php to read real quotes from published authors. 